\title{
Dispersión endozoocórica de plantas en un bosque de encino de Tlaxcala por el cacomixtle (Bassariscus astutus)
}

\author{
Endozoochoric dispersal of plants in oak forest at Tlaxcala by ringtail cat \\ (Bassariscus astutus)
}

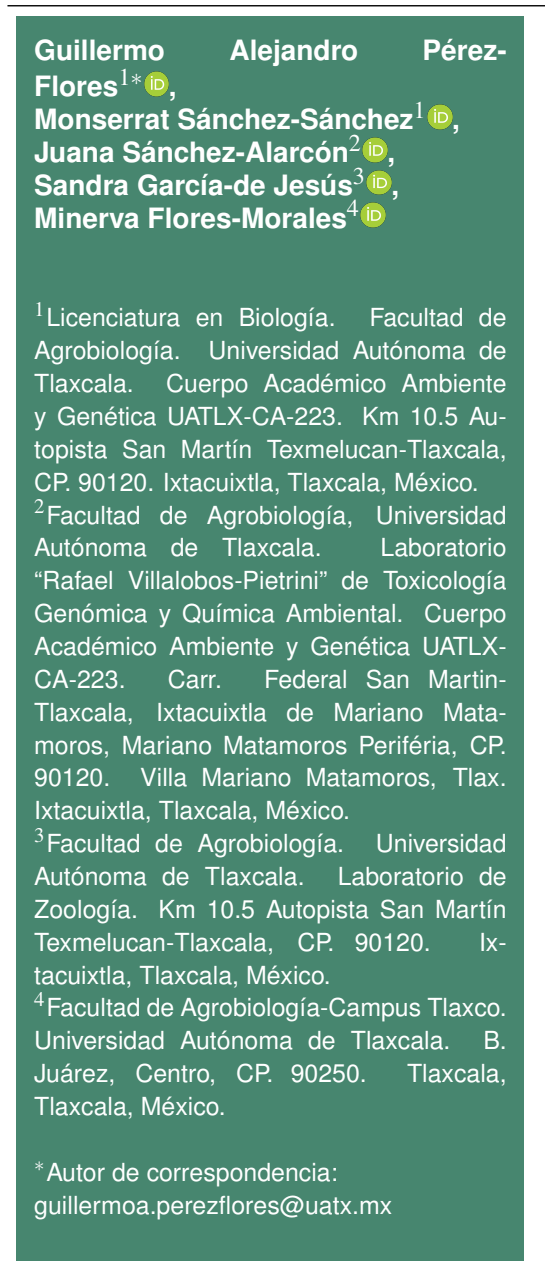

Artículo científico

Recibido: 12 de enero 2021

Aceptado: 20 de noviembre 2021

Como citar: Pérez-Flores GA, SánchezSánchez M, Sánchez-Alarcón J, Garcíade Jesús S, Flores-Morales M (2021) Dispersión endozoocórica de plantas en un bosque de encino de Tlaxcala por el cacomixtle (Bassariscus astutus). Ecosistemas y Recursos Agropecuarios 8(3): e2793. DOI: $10.19136 / \mathrm{era} . \mathrm{a} 8 \mathrm{n} 3.2793$
RESUMEN. La dispersión de semillas es un proceso ecológico que causa efectos en la estructura de las comunidades vegetales para el mantenimiento de su diversidad. El paso de las semillas por el tracto digestivo de algunos vertebrados tiene efectos evidentes en la germinación, el mamífero Bassariscus astutus incluye frutos en su dieta omnívora, lo que se ha documentado en diversos ecosistemas, y presenta una marcada tolerancia a la perturbación. Con el objetivo de evaluar los efectos de la dispersión de cuatro especies de plantas por $B$. astutus, se localizaron, colectaron y procesaron las excretas encontradas en letrinas. Las semillas se recuperaron, identificaron y sometieron a pruebas de viabilidad y germinación, además, se cuantificó la germinación y establecimiento en letrinas. En 76 excretas de 35 letrinas se encontraron semillas de las plantas Juniperus deppeana, Phytolacca icosandra, Opuntia depressa y Amelanchier denticulata. Los resultados mostraron efectos positivos de la dispersión en $O$. depressa y $J$. deppeana con mayor porcentaje de viabilidad y germinación cuando provinieron de endozoocoria, sin efectos en $P$. icosandra y $A$. denticulata. En las letrinas sólo $J$. deppeana y $O$. depressa, parecen ser beneficiadas por la endozoocoria y las condiciones del sitio. Se evidenció el efecto de la dispersión por $B$. astutus en la germinación y reclutamiento de plantas de un bosque templado, es importante considerar el mantenimiento de este proceso en el sitio.

Palabras clave: Cacomixtle, Juniperus, Opuntia, germinación, establecimiento.

ABSTRACT. Seed dispersal is an ecological process that causes effects on plant communities structure to maintenance of their diversity. The passage of seeds through the digestive tract of some vertebrates has evident effects on germination, the mammal Bassariscus astutus includes fruits in its omnivorous diet, this has been documented in various ecosystems, and has a marked tolerance to disturbance. With aim to evaluate the effects of the dispersal of four plant species by $B$. astutus, feces found in latrines were located, collected and processed. Seeds were recovered, identified and subjected to viability and germination tests, in addition, germination and establishment in latrines were quantified. Seeds of plants Juniperus deppeana, Phytolacca icosandra, Opuntia depressa and Amelanchier denticulata were found in 76 feces from 35 latrines. The results showed positive effects of dispersion on $O$. depressa and $J$. deppeana with a higher percentage of viability and germination when they came from endozoocoria, without effects on $P$. icosandra and $A$. denticulata. In the latrines, only $\mathrm{J}$. deppeana and $O$. depressa appear to be benefited by endozoocoria and the site conditions. The effect of dispersal by $B$. astutus on the germination and recruitment of plants from a temperate forest was evidenced; it is important to consider maintenance of this process at the site.

Key words: Ringtail cat, Juniperus, Opuntia, germination, establishment. 


\section{INTRODUCCIÓN}

La dispersión de semillas es un proceso ecológico que causa efectos en la estructura de las comunidades vegetales, en el mantenimiento de su diversidad (Levine y Murrel 2003, Beckman y Rogers 2013) y en la distribución, dinámica y estructura genética de sus poblaciones (Jordano 2007, Nathan et al. 2008). A través de la endozoocoria los vertebrados funcionan como vínculos móviles entre y dentro de parches de bosque, también tienen efectos en la proporción de plantas dispersadas (Karimi et al. 2020). Se ha documentado que el paso de las semillas por el tracto digestivo de los vertebrados tiene efectos en la germinación (Traveset y Verdú 2002), debido a la escarificación mecánica y química provista por el movimiento de los órganos y los jugos gástricos que actúan sobre las semillas y permite acelerar la germinación (Soltani et al. 2018, Costea et al. 2019) así como beneficios en el establecimiento de plántulas y en el reclutamiento de nuevos individuos (Chen et al. 2014, Razafindratsima y Dunham 2015, Palma et al. 2021). También se ha propuesto el impacto de la dispersión en la regeneración de hábitats (Escribano-Ávila et al. 2014, Suárez-Esteban et al. 2013, Salgueiro et al. 2019), lo que es de importancia para entender la resiliencia y susceptibilidad de los ecosistemas ante los fenómenos socio-ambientales actuales como el cambio climático, cacería de dispersores, especies invasoras, deforestación y fragmentación (González-Varo et al. 2013).

En mamíferos, la dispersión de semillas de plantas por endozoocoria es un evento comúnmente registrado (Karimi et al. 2020) así como su consecuente efectividad como dispersores (Torres et al. 2020). En bosques templados, los mesocarnívoros son los principales dispersores (Rubalcava-Castillo et al. 2020), destaca el grupo de los prociónidos entre los que se encuentra Bassariscus astutus o cacomixtle, mamífero nocturno con distribución amplia en América (Harrison 2013, Autin 2021). Es un omnívoro tolerante a los efectos de la fragmentación de los bosques y la urbanización (Castellanos y List 2005, Harrison 2012). La composición de su dieta incluye múltiples especies vegetales, además de las presas diversas, sugiere que es un omnívoro oportunista y generalista (Castillo-Picazo y García-Collazo 2019, Cisneros-Moreno y Martínez-Coronel 2019) que actúa como dispersor de plantas y componente importante en los ecosistemas (Ackerson y Harveson 2006, Stricklan et al. 2020).

Los bosques de encino albergan una gran cantidad de diversidad faunística y florística que se ha ido perdiendo (Romero et al. 2015), están constituidos por varias especies de Quercus (Rzedowski 1978), pero presentan también Abies, Alnus, Arbutus, Buddleja, Cercocarpus, Cupressus, Fraxinus, Garrya, Juniperus, Junglans, Pinus, Platanus, Populus, Prunus, Pseudotsuga y Salix. En el estado de Tlaxcala los encinares ocupan aproximadamente el $5 \%$ de la superficie estatal, distribuidos principalmente sobre cerros y lomeríos en la porción central y norte de la entidad, así como en el Parque Nacional La Malinche, entre los 2200 y 3000 msnm (SEMARNAT-CONAFOR 2015). Las interacciones ecológicas en los bosques de encino de Tlaxcala han sido poco exploradas y la información sobre procesos ecológicos en estos sitios aún es incompleta (Martínez et al. 2007), por lo que se requieren trabajos que indaguen sobre las características de los dispersores, las consecuencias de la dispersión y sus alteraciones antropogénicas con la finalidad de valorar sus posibles efectos en la persistencia de las poblaciones de plantas y en la diversidad (Beckman y Rogers 2013, Beckman et al. 2020). Por lo anterior, e objetivo de esta investigación fue evaluar los efectos de la dispersión por endozoocoria del cacomixtle ( $B$. astutus), en cuatro especies de plantas de un bosque de encino de la Trinidad Tenexyecac en Ixtacuixtla, Tlaxcala.

\section{MATERIALES Y MÉTODOS}

\section{Área de estudio}

La Trinidad Tenexyecac se localiza en el municipio de Ixtacuixtla de Mariano Matamoros del Estado de Tlaxcala México. El bosque pertenece a un predio de propiedad comunal denominada "El Ocotal" localizado en $98^{\circ} 19^{\prime} 23.11^{\prime \prime}$ LN $19^{\circ} 21^{\prime} 15.85$ " LO y 
está a 2479 msnm (Figura 1). El suelo es Fluvisol arenoso limoso, con sedimentos fluviales y también Litosol y alto contenido de carbonato (Werner 1988). El clima es templado subhúmedo con lluvias en los meses de mayo a septiembre. La precipitación media anual es de 500 a $700 \mathrm{~m}$, con temperatura promedio anual de 13 a $17^{\circ} \mathrm{C}$. Por su ubicación geográfica y clima, corresponde una vegetación compuesta principalmente por bosque de Juniperus, el cual en la mayoría de los casos se encuentra fuertemente perturbado o bien ha sido desplazado por la agricultura. Es muy común encontrar este bosque, asociado a elementos propios del matorral xerófito y bosque de encino (INAFED-SG 2010).

\section{Localización de letrinas, colecta y procesamiento de excretas}

Para localizar las letrinas, de febrero a abril de 2017 se realizaron ocho transectos de $10 \times 800 \mathrm{~m}$ en el sitio de estudio con la finalidad de abarcar la mayor área de búsqueda, la ubicación de cada letrina se registró con un GPS (Garmin GPS-Map 64). Para asegurar que las letrinas estuvieran en uso, se realizó un recorrido y se removieron las excretas, dos semanas después se realizó el mismo recorrido para recolectar las excretas en bolsas de plástico de 500 $\mathrm{g}$, se registró la ubicación y las características del sitio de las letrinas como altura del montículo, porcentaje de la pendiente, condición de luz (sol o sombra) y distancia en metros entre letrinas. Para confirmar la presencia de $B$. astutus. Se instalaron tres trampas de arena para huellas de $1 \times 1 \mathrm{~m}$ cerca de las letrinas, se utilizaron trozos de calamar crudo como cebo. La identificación de las excretas en la letrina y de las huellas en las trampas se realizó con el manual para rastreo de mamíferos silvestres de Aranda (2012). En el sitio, se colectaron partes aéreas de plantas con flores y frutos, las muestras se colocaron en una prensa botánica para su posterior identificación, los frutos se utilizaron para comparar sus semillas con las que se recuperaron en las excretas.

Para extraer y separar las semillas de la excreta se utilizó la técnica propuesta por Korschgen (1987) que consiste en (1) Secado para lo cual se colocaron las excretas en bolsas de papel, en una secadora eléctrica para material botánico, a $30^{\circ}$ $\mathrm{C}$, durante $8 \mathrm{~h}$. Después del secado, se almacenaron en bolsas plásticas en refrigeración a $4^{\circ} \mathrm{C}$ para luego continuar con su procesamiento. (2) Lavado y tamizado, para lo cual se colocó la excreta en un cuadro de tela de nylon y licra (malla fina elástica) de $20 \times 20 \mathrm{~cm}$, se cerró con una liga y se sometió a enjuague con fricción en una solución de jabón neutro e hipoclorito de sodio al $1 \%$ para eliminar la mayor cantidad de materia fecal. Posteriormente, el residuo de cada muestra se colocó en un vaso de precipitado de $250 \mathrm{~mL}$, al que se le agregó agua destilada hasta que la muestra fuera cubierta en su totalidad y con ayuda de una varilla de vidrio se agitó hasta que se observó la disgregación. La suspensión se virtió en tamices metálicos de 2 y $0.5 \mathrm{~mm}$ de malla, los cuales se colocaron dentro de una bandeja de plástico siguiendo el orden de mayor a menor tamaño de malla. Las semillas recuperadas se agruparon por similitud de forma y tamaño sobre papel secante y cuantificaron, también se separaron los residuos de origen animal por tipo. (3) Identificación de especies de plantas, se realizó por medio de los frutos de las plantas que se colectaron en la zona, a los cuales se les extrajeron las semillas por lavado de la pulpa con agua sobre tamices, las semillas se compararon con las de las excretas en el microscopio estereoscópico, y se determinó la planta de la que provenía con el material botánico colectado de acuerdo con las características descritas por Rzedowski y Rzedowski (2005) mientras que para las cactáceas se fotografiaron ejemplares con flor y fruto y se determinaron con base en Bravo-Hollis (1978) y Guzmán et al. (2003).

\section{Viabilidad y germinación}

Para evaluar la viabilidad y germinación de las semillas se consideraron los tratamientos de semillas obtenidas de los frutos recolectados de plantas en el sitio (sin procesamiento) y semillas obtenidas por endozoocoria por $B$. astutus (procesadas). Para el primer tratamiento, se eliminó la pulpa del fruto de forma manual por enjuague con agua corriente en un tamiz, para luego secar las semillas al sol sobre papel secante por una semana por periodos de $8 \mathrm{~h}$, y luego durante cinco días se realizó inmersión en agua desti- 


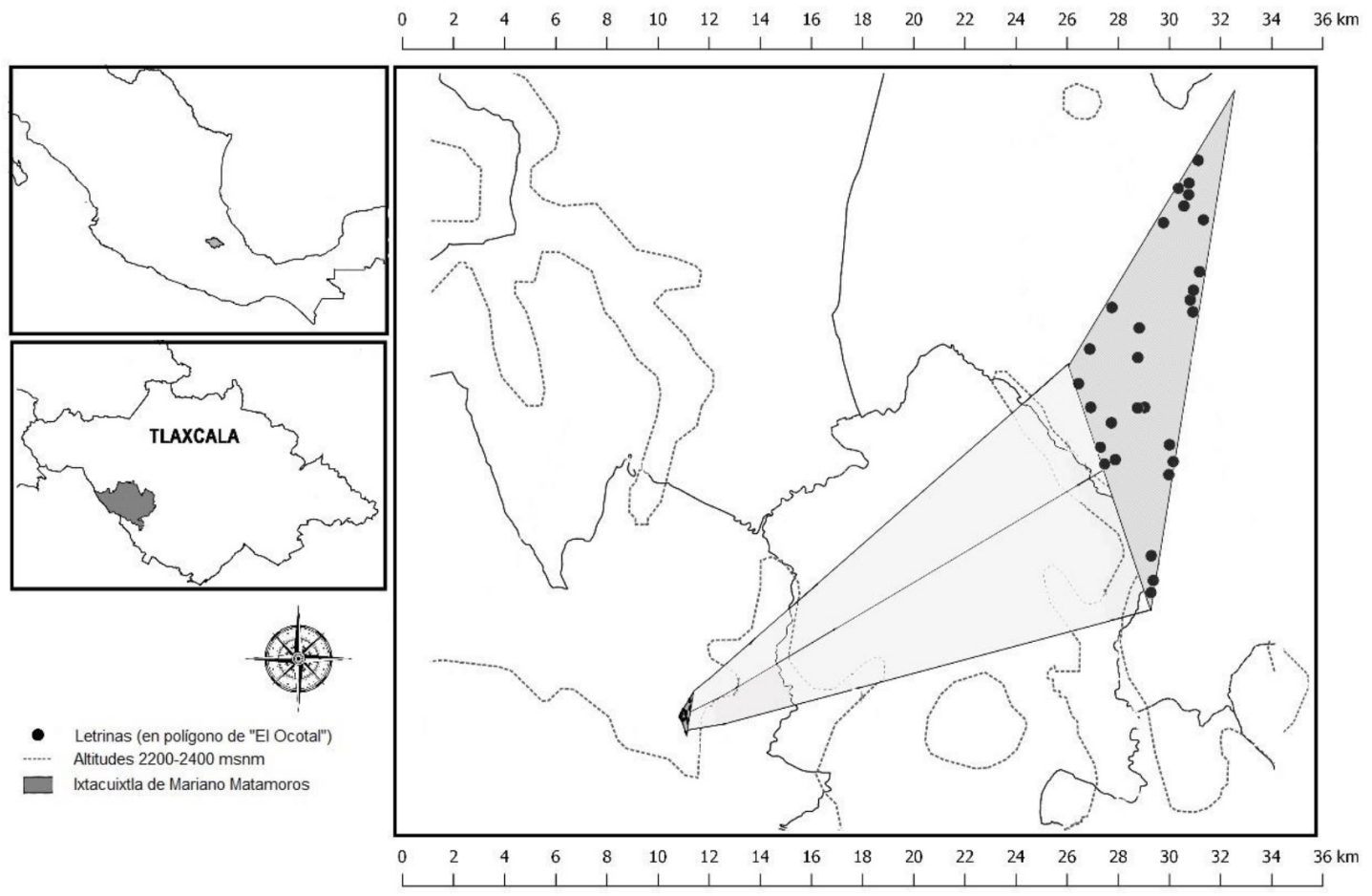

Figura 1. Localización geográfica del sitio de estudio en México y en Tlaxcala (izquierda). El polígono proyectado representa el área de 60 ha del bosque "El Ocotal" los puntos representan la localización de letrinas de $B$. astutus.

lada por $2 \mathrm{~h}$ y secado al sol por $8 \mathrm{~h}$, con la finalidad de simular las condiciones del ambiente que favorecen la escarificación de las semillas (Zamora-Serrano et al. 2012). Mientras que las semillas procesadas que se obtuvieron del proceso de lavado de las excretas se secaron al sol por $8 \mathrm{~h}$ y se mantuvieron en papel secante a la sombra.

Para determinar la viabilidad se utilizó el método de flotación que consiste en colocar las semillas en agua destilada en vasos de precipitado de $250 \mathrm{~mL}$ agitando durante cinco minutos y dejando en reposo 15 min después de los cuales se eliminaron las semillas que flotaron en el agua por considerarse sin embrión viable. Para ambos tratamientos se seleccionaron grupos de 100 semillas de cada especie las cuales se sumergieron en una solución de hipoclorito de sodio al $1 \%$ y se agitaron durante cinco minutos para su desinfección, después de los cuales se enjuagaron con agua destilada en un colador. Posteriormente, se mantuvieron en agua destilada por 48 h con agitación periódica cada 2 h para hidratar la testa. Las semillas se colocaron en cajas Petri con algodón, dentro de recipientes de plástico transparente con capacidad de $500 \mathrm{~mL}$ con tapa, en cada recipiente se colocaron $200 \mathrm{~mL}$ de agua purificada, en su interior se colocó una malla de plástico de $0.5 \mathrm{~mm}$ de abertura y un rectángulo de tela de fieltro previamente esterilizada en contacto con el algodón de la caja en uno de sus extremos y en el otro con el agua para que la tela absorbente mantuviera la humedad constante sobre las semillas; para evitar abrir y cerrar los recipientes para re-hidratar las semillas en el algodón y de esa forma disminuir la probabilidad de contaminación por hongos. Se introdujeron en una cámara de crecimiento vegetal (Shell Lab, modelo L115) hasta la germinación a temperatura constante de $30^{\circ}$ y ciclo luz-oscuridad de $12 \mathrm{~h}$, el tiempo máximo de registro de germinación fue de 54 días.

\section{Germinación y establecimiento en letrinas}

Para evaluar el efecto de la dispersión in situ, 
se cuantificaron las plántulas de reciente germinación, por especie, así como las plantas con altura mayor a $10 \mathrm{~cm}$ establecidas en el perímetro de la letrina, en un radio de 1 metro alrededor. Se determinó la distancia entre plántulas, plantas y letrinas, así como entre plantas establecidas y plantas adultas o conespecíficos más cercanos. Se midió la altura de las plantas con un vernier, la pendiente en porcentaje con un clinómetro, y se registró la condición de luz (sol/sombra) de la letrina, así como la profundidad de la capa de arena fina que se acumula sobre el tepetate, en la cual se acumulan sedimentos.

\section{Análisis estadísticos}

Los resultados se representaron utilizando el promedio y error estándar, así como porcentajes. Para evaluar los porcentajes de viabilidad y germinación se realizaron análisis de varianza (ANOVA) bifactoriales, en donde el tratamiento representó un factor (sin procesamiento y endozoocoria) y la especie de planta el segundo factor, los datos en forma de proporción $(X / 100)$ se transformaron con la función arcoseno para ajustar los datos a los supuestos paramétricos de la prueba. Las comparaciones entre plántulas y plantas de J. deppeana y plántulas por especie en las letrinas se realizaron con pruebas t-student para datos independientes y la comparación de condiciones del ambiente de la letrina (altura, porcentaje de la pendiente y profundidad de la capa de arena) se realizó con pruebas no paramétricas de Mann-Whitney y pruebas de bondad de ajuste de Chi cuadrada. La correlación entre las variables: número de arbolitos, y altura con distancia arbolitoletrina y distancia arbolito-árbol adulto con-específico se verificó con pruebas de correlación de Pearson (Zar 2010).

\section{RESULTADOS}

\section{Letrinas, excretas y plantas identificadas}

Se localizaron 35 letrinas, en la cuales se colectaron 76 excretas. El $55 \%$ de las letrinas se encontraron al nivel del suelo, las demás se encontraron sobre un montículo a una altura sobre el suelo de $23.91 \pm 4.96 \mathrm{~cm}(\bar{X} \pm$ Error estándar). La pendiente promedio del terreno en el que se encontraron fue de $23.59 \pm 3.27 \%$, el $60 \%$ se encontró en lugares despejados con incidencia directa de los rayos solares, mientras que el $40 \%$ fue bajo la sombra de los árboles. La distancia promedio entre letrinas fue de $109.70 \pm 21.41 \mathrm{~m}$, en cada letrina se encontraron de dos o tres excretas, sin excretas de otros mamíferos.

El peso promedio de las excretas después de secado fue de $12.22 \pm 0.97 \mathrm{~g}$. Del total de excretas, $52(68.4 \%)$ tuvieron materia vegetal constituida por semillas y materia de origen animal como exoesqueletos, plumas, pelo y huesos. En tanto que el 23 de las excretas (30.3\%) tuvieron exclusivamente materia vegetal (semillas, fibras, cáscaras) y sólo una excreta (1.3\%) contenía material animal. Del peso de las excretas, $38 \%$ estuvo constituido por material vegetal y $1 \%$ por componentes de origen animal, estos restos en su conjunto representaron materia no digerida con tamaño mayor a $0.5 \mathrm{~mm}$ que fue posible recuperar en un tamiz. Se determinaron las especies vegetales de Juniperus deppeana, Phytolacca icosandra, Opuntia depressa y Amelanchier denticulata.

Las semillas de $P$. icosandra se encontraron en el $94.7 \%$ (72) de las excretas, $A$. denticulata en el $63.1 \%$ (48), Juniperus deppeana en el $60.5 \%$ (46) y 0 . depressa en el $34.2 \%$ (26). En la observación al microscopio estereoscópico se detectó que algunas semillas de $A$. denticulata presentaron perforaciones, que posiblemente pueden ser de insectos. Mientras que $P$. icosandra presentó la mayor cantidad de semillas cuantificadas en cada excreta ( $\bar{X} \pm$ ES), 530 \pm 100 , para $A$. denticulata fue del $22 \pm 5$, para $J$. deppeana de $16 \pm 6$ semillas y para $O$. depressa de $9 \pm 4$ semillas.

\section{Viabilidad y germinación}

Las semillas de $O$. depressa y de $J$. deppeana presentaron los mayores porcentajes de viabilidad (Tabla 1). El análisis de varianza no mostró diferencias significativas entre los tratamientos de semillas sin procesamiento y semillas procesadas (ANOVA $\mathrm{F} 1,61=0.7795, \mathrm{P}=0.3808)$, ni en la interacción tratamiento por especies $(\mathrm{F} 3,61=1.122, \mathrm{P}=$ 
Tabla 1. Comparación de los porcentajes de viabilidad y la germinación en semillas extraídas de los frutos (sin procesamiento) y obtenidas de las excretas de B. astutus (endozoocoria) de cuatro especies de plantas.

\begin{tabular}{lcccc}
\hline & \multicolumn{2}{c}{ Viabilidad (\%) } & \multicolumn{2}{c}{ Germinación (\%) } \\
Especie & Sin procesamiento & Endozoocoria & Sin procesamiento & Endozoocoria \\
\hline J. deppeana & $89.03 \pm 5.623$ & $94.95 \pm 1.879$ & $11.4 \pm 0.4957$ & $38.67 \pm 2.364^{*}$ \\
P. icosandra & $71.73 \pm 2.207$ & $72.33 \pm 1.115$ & $68.25 \pm 4.139$ & $72.67 \pm 2.582$ \\
O. depressa & $98.39 \pm 0.9888$ & $98.15 \pm 0.788$ & $40.38 \pm 4.606$ & $80 \pm 4.082^{*}$ \\
A. denticulata & $12.50 \pm 3.227$ & $5.75 \pm 1.750$ & $6.6 \pm 2.97$ & $0.0 \pm 0.0$ \\
\hline
\end{tabular}

$0.3472)$; mientras que para la viabilidad fue significativamente diferente entre las especies (F3, $61=$ 180.1, $\mathrm{P}<0.0001)$. Las semillas con procesamiento por endozoocoria presentaron mejor germinación, con diferencias significativas entre tratamientos de semillas $(F 1,56=27.56, P<0.0001)$, entre especies $(F 3,56=103.8, P<0.0001)$ y la interacción tratamiento por especies $(F 3,56=19.02, P<0.0001)$, mientras que las pruebas post-hoc demostraron que esta condición la presentaron las especies de $\mathrm{J}$. deppeana y 0 . depressa (Figura 2).

\section{Germinación y establecimiento en letrinas}

En 28 de las 35 letrinas (80\%) se encontraron plántulas de reciente germinación de $J$. deppeana y $O$. depressa, pero no se encontraron plántulas de $P$. icosandra o A. denticulata. En 19 de las 35 letrinas (54\%) se encontraron plántulas de J. deppeana en promedio de $7 \pm 1$ plántulas distribuidas a $0.814 \pm$ $1.33 \mathrm{~m}$ de la letrina y a $12.08 \pm 2.29 \mathrm{~m}$ del árbol con específico adulto más cercano. El número de plantas establecidas de $J$. deppeana en la proximidad de la letrina fue de $4 \pm 0.88$ en 11 letrinas, con altura de $17.93 \pm 1.19 \mathrm{~cm}$. La pendiente en la que se localizaron fue de $19.52 \pm 4.32 \%$ y la profundidad de la capa de arena de $6.45 \pm 1.42 \mathrm{~mm}$. La cantidad de plántulas fue mayor que la de plantas establecidas $(\mathrm{t}=2.833, \mathrm{gl}=42, \mathrm{P}=0.007)$. De $O$. depressa se encontraron $6 \pm 1$ plántulas en 16 letrinas (45.7\%) que tuvieron a $0.424 \pm 0.72 \mathrm{~m}$ de la letrina y a 5.90 $\pm 1.06 \mathrm{~m}$ de la planta adulta de la misma especie. La pendiente en que se localizaron fue de $20.84 \pm$ $2.50 \%$ y la profundidad de la capa de arena de 8.97 $\pm 1.48 \mathrm{~mm}$. La comparación entre el número de plántulas por especie no mostró diferencias $(t=0.5294$, $\mathrm{gl}=34, \mathrm{P}=0.6)$. Para $O$. depressa no se encon- traron plantas en evidente el desarrollo intermedio entre plántulas y adulto. Los adultos de $J$. deppeana y $O$. depressa estuvieron formando parte de la vegetación del bosque de Quercus.

Al comparar las condiciones altura del montículo, exposición al sol, pendiente y profundidad de capa de arena entre las letrinas que presentaron plántulas o plantas establecidas y las que no las tuvieron no se encontraron diferencias estadísticamente significativas en ninguna de las comparaciones (Tabla 2). Al verificar las correlaciones entre el número de plantas establecidas de $\mathrm{J}$. deppeana y las mediciones se encontró correlación negativa con la distancia entre la planta y la letrina, es decir a menor distancia, mayor número de plantas establecidas $(r=0.7110, P=0.0142)$. Entre el número de plantas y la distancia hasta el árbol adulto conspecífico, la correlación no fue significativa $(r=-0.3275, P$ $=0.3255)$ ni entre la altura de los árboles jóvenes y la distancia hasta el árbol adulto $(r=-0.3246, P=$ 0.3301).

\section{DISCUSIÓN}

\section{Letrinas, excretas y plantas identificadas}

Se registró un número de excretas en tres meses poco usual que podría estar relacionado con la densidad poblacional de $B$. astutus, y/o la ausencia de otros carnívoros dispersores. En el Parque Nacional La Malinche se ha registrado la presencia de carnívoros como Urocyon cineroargenteus y $\mathrm{Ca}$ nis latrans (Ruiz-Soberanes y Gómez-Álvarez 2010). Los cuales son dispersores y pueden compartir el es pacio de marcaje fecal como lo reporta RubalcavaCastillo et al. (2020) en el que la cantidad de excretas 

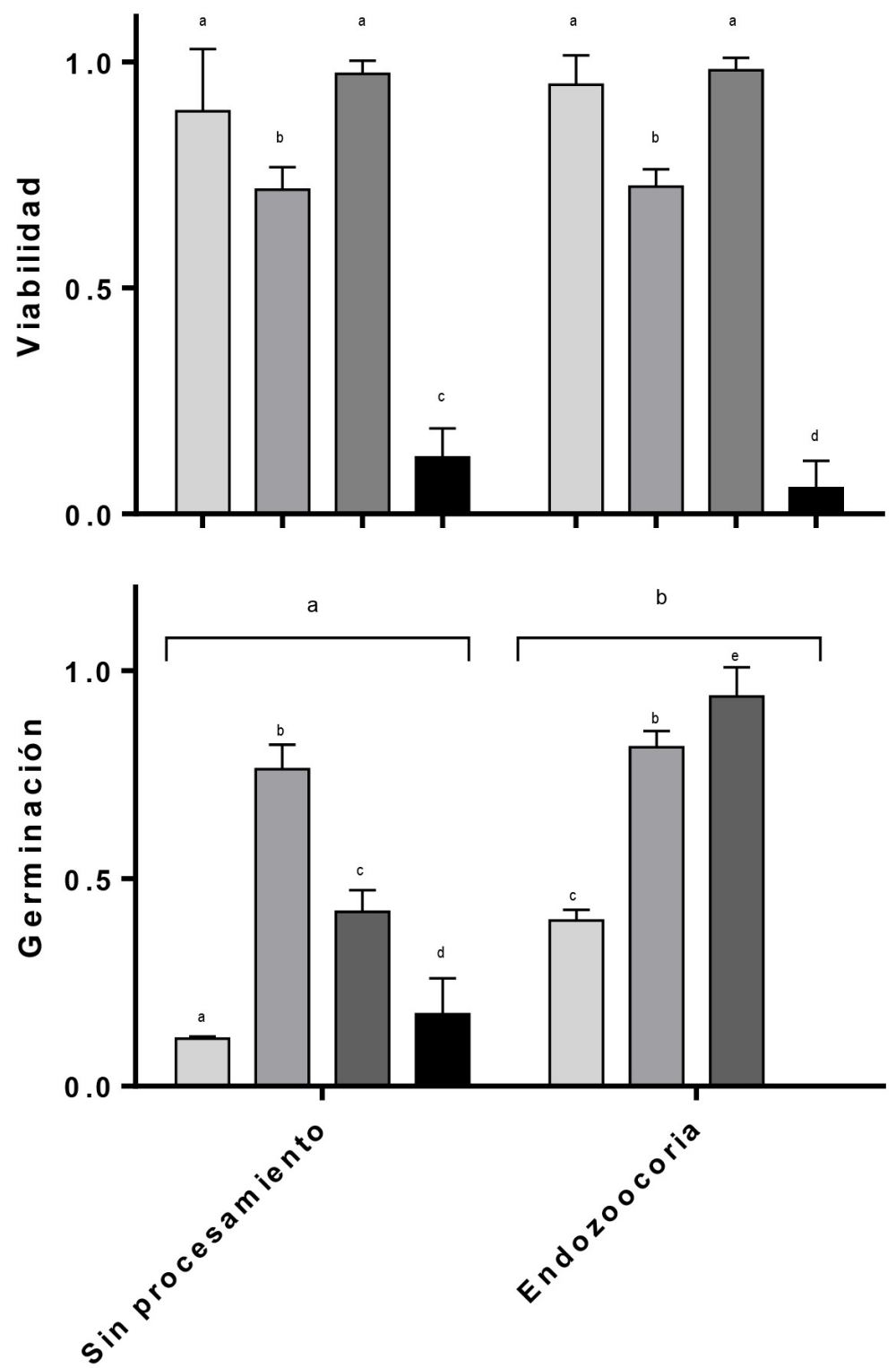
$\square$ J. deppeana
$P$. icosandra
O. depressa
A. denticulata

Figura 2. Viabilidad y germinación de semillas de cuatro especies de plantas (J. deppeana, P. icosandra, O. depressa y A. denticulata) en dos tratamientos: Sin procesamiento a las semillas y endozoocoria provista por $B$. astutus. Los datos del eje $Y$ representan el porcentaje (x100) y las letras diferentes sobre las columnas representan diferencias significativas, se graficaron los promedios de los datos no transformados y las barras de error corresponden a los errores estándar.

colectadas de $B$. astutus en un periodo bianual fue menor (12) que la reportada para $U$. cineroargenteus y $C$. latrans. La disposición espacial de las letrinas parece relacionarse con la conducta de marcaje de $B$. astutus determinada por la selección no aleatoria de sitios a los que los individuos regresan constantemente y utilizan como estrategia de separación de territorios entre individuos (Barja y List 2006), lo que se relaciona con los patrones de dispersión que un organismo puede fomentar en un sitio (Stricklan et al. 2020).

La presencia simultánea de los componentes vegetales y animales en la mayoría de las excretas se ha confirmado para bosques templados y matorral 
Tabla 2. Comparación de las condiciones del ambiente entre las letrinas que presentaron plántulas de las especies J. deppeana y $O$. depressa y las letrinas que no presentaron plántulas. Se presentan promedios \pm error estándar y los valoresestadísticos correspondientes ( $U$ = Mann-Whitney y $\chi^{2}=$ Chi cuadrada).

\begin{tabular}{lccc}
\hline Condición/tipo de letrina & Sin plántulas & Con plántulas & Valores estadísticos \\
\hline Altura de montículo $(\mathrm{cm})$ & $7.857 \pm 7.85$ & $12.82 \pm 5.07$ & $\mathrm{U}=83, \mathrm{P}=0.5316$ \\
Porcentaje de letrinas expuestas a la luz directa del sol $(\%)$ & $43 \pm 20$ & $64 \pm 9.2$ & $\chi^{2}=1.842$, g.l. $=1, \mathrm{P}=0.1747$ \\
Porcentaje de pendiente $(\%)$ & $17.47 \pm 2.27 \%$ & $21.23 \pm 2.36 \%$ & $\chi^{2}=0.1310, \mathrm{~g} . \mathrm{l}=1, \mathrm{P}=0.3174$ \\
Profundidad de capa de arena $(\mathrm{mm})$ & $6.429 \pm 0.922$ & $9.768 \pm 1.55$ & $\mathrm{U}=77.5, \mathrm{P}=0.3881$ \\
\hline
\end{tabular}

xerófilo, por lo que los resultados confirman que los frutos de las plantas forman parte importante de la dieta de $B$. astutus. Al respecto, Castellanos y List (2005) encontraron una proporción similar de componentes animales y vegetales, así como CastilloPicazo y García Collazo (2019) en dos tipos de vegetación y Cisneros-Moreno y Martínez-Coronel (2019) en cuanto a frutos y artrópodos como componentes alimenticios principales en poblaciones rurales y urbanas de cacomixtles. La proporción de excretas que contuvieron sólo materia vegetal podría relacionarse con atributos no explorados en esta investigación como la abundancia de frutos de acuerdo con la temporada o con diferencias individuales de forrajeo de la población de $B$. astutus.

Las especies de plantas identificadas a partir de las semillas recuperadas de las excretas de $B$. astutus se han reportado para Juniperus deppeana (Rubalcava-Castillo et al. 2020), Phytolacca icosandra y especies de Opuntia (Castellanos y List 2005, Castillo-Picazo y García Collazo 2019). La especie Amelanchier denticulata no ha sido reportada como parte de la dieta de B. astutus, pero Auger et al. (2002) reportaron el consumo de frutos de la planta Amelanchier alnifolia por osos negros con las características distintivas de la dispersión al procesarlas a través de su tracto digestivo, lo cual sugiere que los frutos de este género pueden ser elegibles y eventualmente dispersadas por carnívoros. La proporción diferenciada de las semillas en las excretas parece relacionarse con características propias de los frutos como el tamaño, la disponibilidad en la planta, el número de semillas, y las características de $B$. astutus que le permiten manejar y engullir los diferentes frutos, lo que en conjunto forman parte de los rasgos que determinan el tipo de interacción entre planta y dispersor (Dehling et al. 2016, Sebastián-González et al. 2017).

\section{Viabilidad y germinación}

Los resultados de viabilidad que presentaron las semillas de J. deppeana, P. icosandra, O. depressa y $A$. denticulata sugieren que el paso a través del tracto digestivo de $B$. astutus no las afecta de forma significativa como se ha reportado (Auger et al. 2002), para $A$. denticulata se conjetura que la baja viabilidad presentada en ambos tratamientos se relaciona con la observación de perforaciones en las semillas características de daño por insecto (Xu et al. 2015), por lo que se requieren estudios para determinar qué especie de insecto accede a las semillas aún adentro del fruto y el posible efecto en la reproducción de la planta en el sitio de estudio, ya que se conoce poco de la biología de $A$. denticulata (NúñezColín 2010).

Los efectos de la dispersión sobre la germinación de las semillas de las especies estudiadas se pueden caracterizar como positivos para $O$. depressa y $\mathrm{J}$. deppeana, en las cuales el porcentaje después de la endozoocoria fue dos veces y tres veces mayor al de semillas sin procesamiento, y efecto nulo para $P$. icosandra y $A$. denticulata en las cuales el porcentaje de germinación no fue diferente. Para $O$. depressa y J. deppeana se observa un efecto benéfico de que las semillas pasen a través del tracto digestivo del dispersor (Traveset y Verdú 2002). Por lo que se confirma el efecto positivo de la dispersión por carnívoros en la bioregión neotropical reportado por Torres et al. (2020). Para J. deppeana en condiciones artificiales la germinación observada fue de 16 a $36 \%$ en semillas sin estratificar y de $45 \%$ en semillas estratificadas (CONABIO 1999). El porcentaje de germinación obtenido para esta especie $(38.6 \%)$ es diferente al reportado por Rubalcava- 
Castillo et al. (2020) que fue de $0 \%$ en semillas obtenidas de la excreta de $B$. astutus, pero en las de Lynx rufus obtuvo 12.5\%. El porcentaje de germinación de $O$. depressa no tiene comparación con otras especies de Opuntia, pero Monroy-Vázquez et al. (2017) reportan entre 23 y 33\% para O. streptacantha. Se ha sugerido la importancia del proceso de imbibición de las semillas para que puedan germinar, las condiciones de germinación en laboratorio pudieron proveer de humidificación constante de la testa, después de la escarificación natural por la endozoocoria lo cual en conjunto puede explicar esta diferencia.

\section{Germinación y establecimiento en letrinas}

El efecto benéfico en la germinación de dos especies de plantas se confirmó con los datos obtenidos en la proximidad de las letrinas, para $J$. deppeana las plántulas de germinación reciente y los árboles jóvenes indican que el proceso de dispersión tiene efecto en la germinación y establecimiento. Debido a que es una especie de crecimiento lento (CONABIO 1999), se debe considerarse el seguimiento de la supervivencia de los individuos provenientes de la dispersión por $B$. astutus. Para $O$. depressa no se registraron plantas de tamaño intermedio que sugiera una planta establecida de más de un año, por lo que resulta interesante evaluar los procesos ecológicos post-dispersión. Para $P$. icosandra, de la que no se encontraron plántulas ni plantas establecidas en el radio próximo de la letrina, el tamaño de la semilla puede ser determinante de procesos natural de remoción de las semillas de las excretas antes y después de la germinación por procesos post-dispersión que involucran la dispersión secundaria y la depredación de semillas (Traveset y Verdú 2002, Schupp et al. 2010) como se ha documentado para $P$. americana, en la que los procesos mencionados pueden tener un efecto directo en los patrones de dispersión de sus poblaciones (Orrock et al. 2006). Las condiciones ambientales del bosque de encino del sitio de estudio son de importancia para que los procesos de dispersión-germinación-establecimiento de las plantas sea efectivo, por lo que se recomienda comparar los efectos de la dispersión con los que ocurren en ambientes colindantes. Debido a que se han registrado efectos de la dispersión en la germinación in situ y el establecimiento de una especie de la vegetación nativa, su inclusión en el bosque de encino forma parte de los procesos de intensificación y colonización en parches adyacentes por efecto de la fragmentación (Spennemann 2020). La corta distancia entre plantas establecidas y los árboles de $\mathrm{J}$. deppeana, indica que es una de las especies dispersadas (Cain et al. 2000). También la correlación entre el número de plántulas y la distancia a la letrina indica que la excreta favorece la germinación, en plantas establecidas, su número no se incrementó con la cercanía a los árboles de la misma especie, lo que sugiere que los dispersores realizan movilidad local y que no se trata de plantas que germinaron en la proximidad de la planta madre. La altura de las plantas establecidas tampoco se correlacionó con la distancia hasta el árbol conespecífico como debería haber ocurrido predictiblemente sin la participación de un dispersor (Thomson et al. 2011). Lo que indica dentro del bosque se están favoreciendo procesos de reclutamiento y flujo genético al interior de la población (Jordano 2007), más que el intercambio entre poblaciones por dispersión a mayor distancia (Nathan et al. 2008).

\section{CONCLUSIONES}

La dispersión por endozoocoria que provee el cacomixtle puede tener efectos para algunas especies de plantas en la germinación, el establecimiento, y la regeneración de los relictos de bosques de la región central de México. Los efectos de la dispersión que realiza $B$. astutus para $J$. deppeana pueden tener un papel determinante en su presencia y continuidad del paisaje, por lo que es importante considerar acciones de conservación que incluyan las condiciones para mantener este proceso en los bosques templados.

\section{AGRADECIMIENTOS}

A Ana Minor, Arabella García y Maricarmen Islas por su apoyo en los muestreos, así como René 
Grada por la facilidades en el uso de las instalaciones del Laboratorio de Agroecología y Vida Silvestre. Con especial reconocimiento por su valioso apoyo a los re- visores anónimos que contribuyeron a mejorar el escrito.

\section{LITERATURA CITADA}

Ackerson BK, Harveson LA (2006) Characteristics of a ringtail (Bassariscus astutus) population in Trans Pecos, Texas. Texas Journal of Science 58: 169-184.

Aranda SJM (2012) Manual para el rastreo de mamíferos silvestres de México. Comisión Nacional para el Conocimiento y Uso de la Biodiversidad. México. 255p.

Auger J, Meyer SE, Black HL (2002) Are American black bears (Ursus americanus) legitimate seed dispersers for fleshy-fruited shrubs? The American Midland Naturalist 147: 352-367.

Autin B (2021) LibGuides: North American Ringtail (Bassariscus astutus) Fact Sheet: Distribution \& Habitat. https://ielc.libguides.com/sdzg/factsheets/ringtail/distribution. Fecha de consulta: 31 de octubre de 2021.

Barja I, List R (2006) Faecal marking behaviour in ringtails (Bassariscus astutus) during the non-breeding period: spatial characteristics of latrines and single faeces. Chemoecology 16: 219-222.

Beckman NG, Rogers HS (2013) Consequences of Seed Dispersal for Plant Recruitment in Tropical Forests: Interactions within the seedscape. Biotropica 45: 666-681.

Beckman NG, Aslan CE, Rogers HS, Kogan O, Bronstein JL, Bullock JM, Hartig F, HilleRisLambers J, Zhou Y, Zurell D, Brodie JF, Bruna EM, Cantrell RS, Decker RR, Efiom E, Fricke EC, Gurski K, Hastings A, Johnson JS, Zambrano J (2020) Advancing an interdisciplinary framework to study seed dispersal ecology. AoB PLANTS 12. DOI: 10.1093/aobpla/plz048.

Bravo-Hollis H (1978) Las cactáceas de México. UNAM. México. 743p.

Cain ML, Milligan BG, Strand AE (2000) Long-distance seed dispersal in plant populations. American Journal of Botany 87: 1217-1227.

Castellanos G, List R (2005) Área de actividad y uso de hábitat del cacomixtle (Bassariscus astutus) en el Pedregal de San Ángel. Revista Mexicana de Mastozoología 9: 113-122.

Castillo-Picazo GER, García-Collazo R (2019) Comparación de la dieta del cacomixtle norteño, Bassariscus astutus de un bosque templado y un matorral xerófilo, del centro de México. BIOCYT Biología Ciencia y Tecnología 12: 45-48.

Chen L, Wang L, Baiketuerhan Y, Zhang C, Zhao X, von-Gadow K (2014) Seed dispersal and seedling recruitment of trees at different successional stages in a temperate forest in northeastern China. Journal of Plant Ecology 7: 337-346.

Cisneros-Moreno C, Martínez-Coronel M (2019) Alimentación del cacomixtle (Bassariscus astutus) en un ambiente urbano y uno agrícola en los valles centrales de Oaxaca. Revista Mexicana de Mastozoología (Nueva Época) 9: 31-43.

CONABIO (1999) Fichas de especies. Juniperus deppeana. Comisión Nacional para el Uso y Conocimiento de la Biodiversidad. http://www.conabio.gob.mx. Fecha de consulta: 18 de septiembre de 2019.

Costea M, Miari HE, Laczkó L, Fekete R, Molnár AV, Lovas-Kiss Á, Green AJ (2019) The effect of gut passage by waterbirds on the seed coat and pericarp of diaspores lacking "external flesh": Evidence for widespread adaptation to endozoochory in angiosperms. PLOS ONE 14(12): e0226551. DOI: 10.1371/journal.pone. 0226551 
Dehling DM, Jordano P, Schaefer HM, Böhning-Gaese K, Schleuning M (2016) Morphology predicts species' functional roles and their degree of specialization in plant-frugivore interactions. Proceedings of the Royal Society B: Biological Sciences 283: 20152444. DOI: 10.1098/rspb.2015.2444.

Escribano-Ávila G, Calvino-Cancela M, Pias B, Virgos E, Valladares F, Escudero A (2014) Diverse guilds provide complementary dispersal services in a woodland expansion process after land abandonment. Journal of Applied Ecology 51: 1701-1711.

González-Varo JP, López-Bao JV, Guitián J (2013) Functional diversity among seed dispersal kernels generated by carnivorous mammals. Journal of Animal Ecology 82: 562-571.

Guzmán U, Arias S, Dávila P (2003) Catálogo de Cactáceas mexicanas. UNAM, CONABIO. México. 315p.

Harrison RL (2012) Ringtail (Bassariscus astutus) Ecology and behavior in Central New Mexico, USA. Western North American Naturalist 72: 495-506.

Harrison R (2013) Ringtail (Bassariscus astutus) noninvasive survey methods, density, and occupancy in central New Mexico, USA. Western North American Naturalist 73: 365-372

INAFED-SG (2010) Enciclopedia de los municipios y delegaciones de México. Tlaxcala, Ixtacuixtla de Mariano Matamoros. Instituto para el Federalismo y el Desarrollo Municipal, Secretaría de Gobernación. http://www. inafed.gob.mx. Fecha de consulta: 20 de junio de 2019.

Jordano P (2007) Frugivores, seeds and genes: Analyzing the key elements of seed shadows. In: Dennis AJ, Schupp EW, Green RJ y Westcott DA (eds). Seed dispersal: Theory and its application in a changing world. CAB International. Australia. pp: 229-251.

Karimi S, Hemami MR, Tarkesh Esfahani M, Baltzinger C (2020) Endozoochorous dispersal by herbivores and omnivores is mediated by germination conditions. BMC Ecology 20: 49. DOI: 10.1186/s12898-020-003173.

Korschgen LJ (1987) Procedimiento para el análisis de los hábitos alimentarios. In: Rodríguez TR (ed). Manual de gestión de vida silvestre. Cuarta Edición. The Wildlife Society. Washington, USA. pp: 119-134.

Levine JM, Murrell DJ (2003) The community-level consequences of seed dispersal patterns. Annual Review of Ecology, Evolution, and Systematics 34: 549-574.

Martínez AJ, Lezama-Delgado E, Sainos P, Álvarez A (2007) El tamaño sí importa: los frutos grandes de Juniperus deppeana Steud. (Sabino) son más susceptibles a depredación por insectos. Madera y Bosques 13: 65-81.

McKinney M (2002) Urbanization, biodiversity, and conservation. BioScience 52: 883-890.

Monroy-Vázquez ME, Peña-Valdivia CB, García JR, Solano E, Campos H, García E (2017) Chemical scarification and ozone in seed dormancy alleviation of wild and domesticated Opuntia, Cactaceae. Ozone: Science y Engineering 39: 104-114.

Nathan R, Schurr FM, Spiegel O, Steinitz O, Trakhtenbrot A, Tsoar A (2008) Mechanisms of long-distance seed dispersal. Trends in Ecology and Evolution 23: 638-647.

Núñez-Colín CA (2010) Distribución y caracterización eco-climática del membrillo cimarrón (Amelanchier denticulata (Kunth) Koch) en México. Revista Chapingo Serie Horticultura 16: 195-206.

Orrock JL, Levey DJ, Danielson BJ, Damschen El (2006) Seed predation, not seed dispersal, explains the landscape-level abundance of an early-successional plant. Journal of Ecology 94: 838-845. 
Palma AC, Goosem M, Fensham RJ, Goosem S, Preece ND, Stevenson PR, Laurance SGW (2021) Dispersal and recruitment limitations in secondary forests. Journal of Vegetation Science 32: e12975. DOI: 10.1111/jvs.12975.

Razafindratsima OH, Dunham AE (2015) Assessing the impacts of nonrandom seed dispersal by multiple frugivore partners on plant recruitment. Ecology 96: 24-30.

Romero R, Rojas Z, Rubio L (2015) Encinos de México. UNAM-Facultad de Estudios Superiores Iztacala. México. 250p.

Rubalcava-Castillo FA, Sosa-Ramírez J, Luna-Ruíz JJ, Valdivia-Flores AG, Díaz-Núñez V, Íñiguez-Dávalos LI (2020) Endozoochorous dispersal of forest seeds by carnivorous mammals in Sierra Fría, Aguascalientes, Mexico. Ecology and Evolution 10: 2991-3003.

Ruiz-Soberanes JA, Gómez-Álvarez G (2010) Estudio mastofaunístico del Parque Nacional Malinche, Tlaxcala, México. Therya 1: 97-110.

Rzedowski J (1978) Vegetación de México. Limusa. México. 504p.

Rzedowski GC de J Rzedowski (2005) Flora fanerogámica del Valle de México. 2a. ed. 1a reimp. Instituto de Ecología, Comisión Nacional para el Conocimiento y Uso de la Biodiversidad. Pátzcuaro, Michoacán, México. 1406p.

Salgueiro V, Silva C, Eufrázio S, Salgueiro PA, Vaz PG (2019) Endozoochory of a dry fruited tree aids quarry passive restoration and seed soaking further increases seedling emergence. Restoration Ecology 28: 668678.

Sebastián-González E, Pires MM, Donatti Cl, Guimarães JrPR, Dirzo R (2017) Species traits and interaction rules shape a species-rich seed-dispersal interaction network. Ecology and Evolution 7: 4496-4506.

Schupp EW, Jordano P, Gómez JM (2010) Seed dispersal effectiveness revisited: A conceptual review. New Phytologist 188: 333-353.

SEMARNAT-CONAFOR (2015) Inventario estatal forestal y de suelos. Tlaxcala. Secretaría de Medio Ambiente y Recursos Naturales. Zapopan, Jalisco, México. 130p.

Spennemann DH (2020) Frugivory and seed dispersal revisited: Codifying the plant-centred net benefit of animalmediated interactions. Flora 263: 151534.

Stricklan D, Saud P, Cibils AF, Steiner RL, Cram DS, Young K, Faist AM (2020) Germination of one-seed juniper seeds distributed by different frugivore groups. Rangeland Ecology \& Management 73: 433-440.

Soltani E, Baskin CC, Baskin JM, Heshmati S, Mirfazeli MS (2018) A meta-analysis of the effects of frugivory (endozoochory) on seed germination: Role of seed size and kind of dormancy. Plant Ecology 219: 12831294.

Suárez-Esteban A, Delibes M, Fedriani JM (2013) Barriers or corridors? The overlooked role of unpaved roads in endozoochorous seed dispersal. Journal of Applied Ecology 50: 767-774.

Thomson FJ, Moles AT, Auld TD, Kingsford RT (2011) Seed dispersal distance is more strongly correlated with plant height than with seed mass. Journal of Ecology 99: 1299-1307.

Torres DA, Castaño JH, Carranza-Quiceno JA (2020) Global patterns in seed germination after ingestion by mammals. Mammal Review 50: 278-290. 
Traveset A, Verdú M (2002) A meta-analysis of the effect of gut treatment on seed germination. In: Levey DJ, Silva WR, Galetti M (eds.). Seed dispersal and frugivory: Ecology, evolution and conservation. Third International Symposium-Workshop on Frugivores and Seed Dispersal, CAB International São Pedro, Brazil. pp: 339350.

Werner G (1988) Los suelos en el estado de Tlaxcala. Altiplano Central Mexicano. Gobierno Estatal de Tlaxcala, Universidad Autónoma de Tlaxcala, Centro de Estudios Municipales de Tlaxcala. México. 198p.

Willson MF, Traveset A (2000) The ecology of seed dispersal. In: Fenner M (ed) Seeds: the ecology of regeneration in plant communities. CAB International. Pennsylvania, USA. pp. 85-110.

Xu Y, Shen Z, Li D, Guo Q (2015) Pre-Dispersal Seed Predation in a Species-Rich Forest Community: Patterns and the Interplay with Determinants. PLOS ONE 10: e0143040. https://doi.org/10.1371/journal.pone.01430 40

Zamora-Serrano C, Cruz FJ, López J (2012) Tecnología para la preservación de Juniperus comitana Mart. y J. deppeana var. gambona (Mart.) R. P. Adams. Revista mexicana de Ciencias Forestales 3: 91-98.

Zar JH (2010) Biostatistical analysis. Fifth Edition. Prentice Hall, Inc. New Jersey. 944p. 